\title{
Effects of reflective scattering in nuclear collisions
}

\author{
S.M. Troshin, N.E. Tyurin \\ Institute for High Energy Physics, \\ Protvino, Moscow Region, 142281, Russia
}

\begin{abstract}
We discuss effects of reflective scattering for heavy nuclei collisions and the deconfined matter formation at the LHC and asymptotical energies. Reflective scattering in hadron collisions leads to decreasing matter density with energy beyond the LHC energies. Limiting form of energy dependence of hadron density is obtained.
\end{abstract}




\section{Introduction}

Deconfined state of matter has been discovered by the four major experiments at RHIC [1]. The highest values of energy and density have been reached in these experiments. This deconfined state appears to be strongly interacting collective state with properties of the perfect liquid. The nature of the deconfined form of matter is not known. The importance of the experimental discoveries at RHIC is that the matter is strongly correlated and reveals high degree of the coherence when it is well beyond the critical values of density and temperature.

In this note we address one aspect of the broad problem of transition to the deconfined state of matter, namely, we discuss the role of the reflective scattering on the energy dependence of density in the percolation mechanism of the transition to the deconfined state of matter.

The main idea of the percolation mechanism of deconfinement is a formation in the certain volume of a connected hadron cluster due to increasing temperature and/or hadron density [2], i.e. when vacuum as a connected medium disappears, the deconfinement takes place. This process has typical critical dependence on particle density. Thus, it was proposed to use percolation to define the states of matter and consider the disappearance of a large-scale vacuum as the end of hadronic matter existence [2, 3].

The reflective scattering in its turn is a natural interpretation of the unitarity saturation based on the optical concepts in high energy hadron scattering [4]. At very high energies the elastic scattering can go beyond the black disk limit and become a reflective at small impact parameters, i.e. elastic $S$-matrix gets negative values. The evolution with energy is characterized by increasing albedo due to the interrelated increase of reflection and decrease of absorption at small impact parameters. Asymptotically, picture of particle collisions with small impact parameters resembles hard balls collisions. Therefore, the presence of reflective scattering reduces the available for scattering volume and results in lower densities of hadronic matter needed for triggering percolation mechanism of deconfinement.

\section{Effective degrees of freedom and overlap of hadrons as a mechanism of deconfinement}

For the beginning, we would like to note that there is an interesting possibility that the origin of the transient state in hadron and nuclear collisions and its dynamics along with hadron structure can be related to the mechanism of spontaneous chiral symmetry breaking $(\chi \mathrm{SB})$ in $\mathrm{QCD}[5]$, which leads to the generation of quark 
masses and appearance of quark condensates. This mechanism describes transition of the current into constituent quarks. The gluon field is considered to be responsible for providing quarks with masses and its internal structure through the instanton mechanism of the spontaneous chiral symmetry breaking. Massive constituent quarks appear as quasiparticles, i.e. current quarks and the surrounding clouds of quark-antiquark pairs. Quark radii are determined by the radii of the surrounding clouds.

Collective excitations of the condensate are the Goldstone bosons, and the constituent quarks interact with each other via exchange of the Goldstone bosons; this interaction is mainly due to pion field. Pions themselves are the bound states of massive quarks. For quark-pion interaction the following form can be used [6]:

$$
\mathcal{L}_{I}=\bar{Q}\left[i \not \partial-M \exp \left(i \gamma_{5} \pi^{A} \lambda^{A} / F_{\pi}\right)\right] Q, \quad \pi^{A}=\pi, K, \eta .
$$

This interaction is strong. The general form of the total effective Lagrangian $\left(\mathcal{L}_{Q C D} \rightarrow \mathcal{L}_{e f f}\right)$ relevant for description of the non-perturbative phase of QCD includes the three terms [7]

$$
\mathcal{L}_{\text {eff }}=\mathcal{L}_{\chi}+\mathcal{L}_{I}+\mathcal{L}_{C}
$$

Here $\mathcal{L}_{\chi}$ is responsible for the spontaneous chiral symmetry breaking and turns on first. To account for the constituent quark interaction and confinement the terms $\mathcal{L}_{I}$ and $\mathcal{L}_{C}$ are introduced. The $\mathcal{L}_{I}$ and $\mathcal{L}_{C}$ do not affect the internal structure of the constituent quarks.

The picture of a hadron consisting of constituent quarks embedded into quark condensate implies that overlapping and interaction of peripheral clouds occur at the first stage of hadron interaction. At this stage, part of the effective lagrangian $\mathcal{L}_{C}$ is turned off and transition to the deconfined state of hadron matter occurs.

The mechanism described above [8], when the part of the effective lagrangian $\mathcal{L}_{C}$ is suddenly turning off, can be formulated in terms of percolation theory. As it was already mentioned in the Introduction, it was recently proposed to use this theory as a mechanism of deconfinement in the form of a rapid "cross-over" in [2, 3]. Usually, phase transitions are associated with symmetry breaking and singularities in the partition function. Percolation theories also describe critical behavior, but those consider geometrical quantities such as cluster formation (cf. [2] and references therein). These quantities also diverge but its divergence cannot be obtained from the partition function.

\section{Reflective scattering and percolation}

In [2] the two possibilities for the deconfinement were considered: one is related to the percolation of permeable spheres (mesons) and another one deals with per- 
colation of spheres with repulsive cores (baryons). While the latter is a good approximation for the static case and the case of increasing baryon density, it seems to be not appropriate for the real processes of nuclei collisions. Indeed, the presence of the repulsive core in the structure of baryons is essential for preventing nuclei from collapsing. But, in the processes of nuclear collisions they loose their identity. Therefore, no reasons exist to extend static properties to the dynamics of nuclear interactions and expect presence of the repulsive core in baryons participating in nuclear collisions. Indeed, experimental studies of the hadron elastic and inelastic overlap functions

$$
h_{e l}(s, b) \equiv \frac{d \sigma_{e l}}{d b^{2}}=4 \pi|f(s, b)|^{2} ; h_{\text {inel }}(s, b) \equiv \frac{d \sigma_{\text {inel }}}{d b^{2}}=4 \pi \eta(s, b)
$$

at modern energies demonstrate an absence of the region at small impact parameters where probability of the elastic and/or inelastic overlaps of hadrons (mesons and baryons) is small or zero. Note here, that the unitarity relation written for the elastic scattering amplitude $f(s, b)$ in the impact parameter representation relates it with the contribution of the inelastic channels $\eta(s, b)$ :

$$
\operatorname{Im} f(s, b)=|f(s, b)|^{2}+\eta(s, b) .
$$

The situation is expected to be different at higher energies when inelastic overlap function $h_{\text {inel }}(s, b)$ would have a peripheral $b$-dependence and will tend to zero for $b=0$ at $s \rightarrow \infty$ cf. e.g [4]). As it was pointed out in the Introduction, corresponding behavior of elastic scattering $S$-matrix $S(s, b)$ can then be interpreted as an appearance of a reflecting ability of scatterer due to increase of its density beyond some critical value, corresponding to refraction index noticeably greater than unity. In another words, the scatterer has now not only absorption ability (due to presence of inelastic channels), but it starts to be reflective at very high energies. The appearance of this reflective ability in its turn can be related to the finite size of an extended parton [9]. In central collisions, $b=0$, scattering approaches to the completely reflecting limit $S=-1$ at $s \rightarrow \infty$.

Transition to the reflective scattering mode is naturally reproduced by the $U$ matrix form of elastic unitarization. The elastic scattering $S$-matrix $(2 \rightarrow 2$ scattering matrix element) in the impact parameter representation is written in this unitarization scheme in the form of linear fractional transform:

$$
S(s, b)=\frac{1+i U(s, b)}{1-i U(s, b)}
$$

where $U(s, b)$ is the generalized reaction matrix, which is considered to be an input dynamical quantity similar to an input Born amplitude. For simplicity consider the case of pure imaginary $U$-matrix and make the replacement $U \rightarrow i U$, 
i.e.

$$
S(s, b)=\frac{1-U(s, b)}{1+U(s, b)}
$$

It can easily be seen that reflective scattering mode (when $S(s, b)<0$ ) starts to appear at the energy $s_{R}$, which is determined as a solution of the equation

$$
U\left(s_{R}, b=0\right)=1 .
$$

At $s>s_{R}$ the elastic scattering acquires ability for reflection, while inelastic overlap function $h_{\text {inel }}(s, b)$ gets a peripheral impact parameter dependence in the region $s>s_{R}$. Such dependence is a manifestation of the self-damping of the inelastic channels at small impact parameters. The function $h_{\text {inel }}(s, b)$ reaches its maximum value at $b=R(s)$, while the elastic scattering (due to reflection) occurs effectively at smaller values of impact parameter, i.e. $\left\langle b^{2}\right\rangle_{e l}<\left\langle b^{2}\right\rangle_{\text {inel }}$. Note that

$$
\left\langle b^{2}\right\rangle_{i}=\frac{1}{\sigma_{i}} \int b^{2} d \sigma_{i} \equiv \frac{1}{\sigma_{i}} \int_{0}^{\infty} b^{2} \frac{d \sigma_{i}}{d b^{2}} d b^{2},
$$

where $i=e l$, inel.

At the values of energy $s>s_{R}$ the equation $U(s, b)=1$ has a solution in the physical region of impact parameter values, i.e. $S(s, b)=0$ at $b=R(s)$. This line is shown in the $s$ and $b$ plane in Fig. 1 alongside with the regions where elastic $S$-matrix has positive and negative values. The dependence of $S(s, b)$ on impact

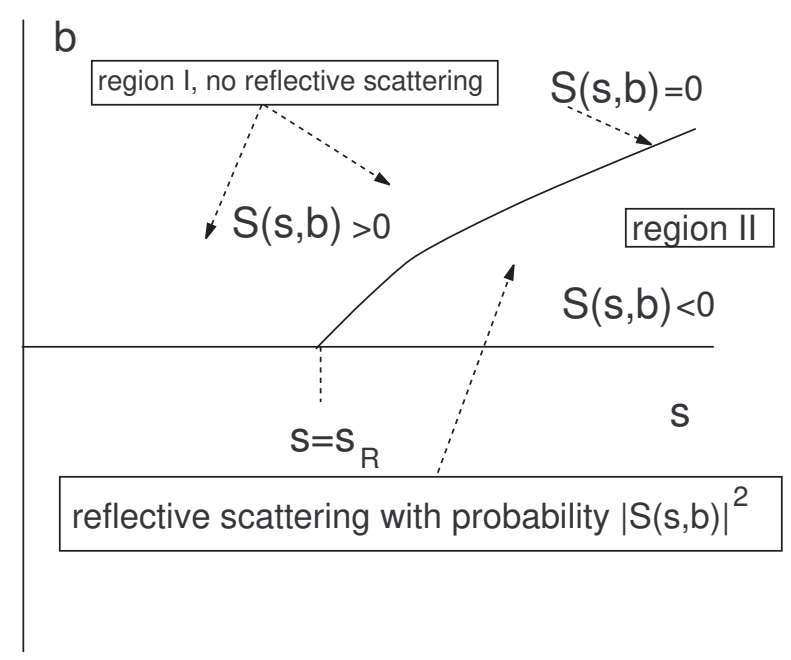

Figure 1: Regions of positive (absorptive scattering) and negative values (absorptive and reflective scattering) of the function $S(s, b)$ in the $s$ and $b$ plane.

parameter $b$ at fixed energies (in the region $s>s_{R}$ ) is depicted on Fig. 2. 


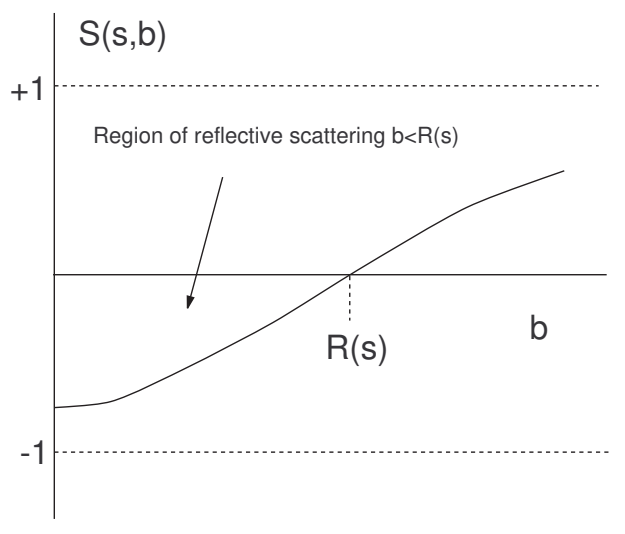

Figure 2: Qualitative impact parameter dependence of the function $S(s, b)$ for the energies $s>s_{R}$.

The probability of reflective scattering at $b<R(s)$ and $s>s_{R}$ is determined by the magnitude of $|S(s, b)|^{2}$; this probability is equal to zero at $s \leq s_{R}$ and $b \geq R(s)$ (region I on Fig.1). The form of $U(s, b)$ depends on the particular model assumptions, but for our qualitative purposes it is sufficient that it increases with energy in a power-like way and decreases with impact parameter like a linear exponent. The dependence of $R(s)$ is determined then by the logarithmic functional dependence $R(s) \sim \frac{1}{M} \ln s$, this dependence is consistent with analytical properties of the resulting elastics scattering amplitude in the complex $t$-plane and mass $M$ can be related to the pion mass.

Thus, at the energies $s>s_{R}$ reflective scattering will mimic presence of repulsive core in hadron and meson interactions as well. Presence of the reflective scattering can be accounted for using van der Waals method (cf. [10]). This approach was used originally for description of the fluids behavior starting from the gas approximation by means of taking into account the nonzero size of molecules.

\section{Effects of the reflective scattering mode for nu- clear collisions}

Consider central collision of two identical nuclei having $N$ hadrons in total with center of mass energy $\sqrt{s}$ per nucleon and calculate hadron density $n_{R}(T, \mu)=$ $N / V$ in the initial state at given temperature $T$ and baryochemical potential $\mu$ in the presence of reflective scattering. The effect of the reflective scattering of hadrons is equivalent to decrease of the volume of the available space which the hadrons are able to occupy in the case when reflective scattering is absent. Thus 
followings to van der Waals method, we must then replace volume $V$ by $V-$ $p_{R}(s) V_{R}(s) \frac{N}{2}$, i.e. we should write

$$
n(T, \mu)=\frac{N}{V-p_{R}(s) V_{R}(s) \frac{N}{2}}
$$

where $n(T, \mu)$ is hadron density without account for reflective scattering and $p_{R}(s)$ is the averaged over volume $V_{R}(s)$ probability of reflective scattering:

$$
p_{R}(s)=\frac{1}{V_{R}(s)} \int_{V_{R}(s)}|S(s, r)|^{2} d^{3} x .
$$

The volume $V_{R}(s)$ is determined by the radius of the reflective scattering. Here we assume spherical symmetry of hadron interactions, i.e. we replace impact parameter $b$ by $r$ and approximate the volume $V_{R}(s)$ by $V_{R}(s) \simeq(4 \pi / 3) R^{3}(s)$. Hence, the density $n_{R}(T, \mu)$ is connected with corresponding density in the approach without reflective scattering $n(T, \mu)$ by the following relation

$$
n_{R}(T, \mu)=\frac{n(T, \mu)}{1+\alpha(s) n(T, \mu)},
$$

where $\alpha(s)=p_{R}(s) V_{R}(s) / 2$. Let us now estimate change of the function $n_{R}(T, \mu)$ due to the presence of reflective scattering. We can approximate $p_{R}(s)$ by the value of $|S(s, b=0)|^{2}$ which tends to unity at $s \rightarrow \infty$. It should be noted that the value $\sqrt{s_{R}} \simeq 2 \mathrm{TeV}$ [11]. Below this energy there is no reflective scattering, $\alpha(s)=0$ at $s \leq s_{R}$, and therefore corrections to the hadron density are absent. Those corrections are small when the energy is not too much higher than $s_{R}$. At $s \geq s_{R}$ the value of $\alpha(s)$ is positive, and presence of reflective scattering diminishes hadron density. We should expect that this effect would already be noticeable at the LHC energy $\sqrt{s} \simeq 5 \mathrm{TeV}$ in $\mathrm{Pb}+\mathrm{Pb}$ collisions. At very high energies $(s \rightarrow \infty)$

$$
n_{R}(T, \mu) \sim 1 / \alpha(s) \sim M^{3} / \ln ^{3} s .
$$

This limiting dependence for the hadron density appears due to the presence of the reflective scattering which results in similarity of head-on hadron collisions with scattering of hard spheres. It can be associated with saturation of the FroissartMartin bound for the total cross-section. It should be noted that this dependence has been obtained under assumption on spherical symmetry of hadron interaction region. Without this assumption, limiting dependence of the hadron density in transverse plane can only be obtained, i.e. transverse plane density of hadrons would have then the following behavior

$$
n_{R}(T, \mu) \sim M^{2} / \ln ^{2} s .
$$




\section{Conclusion}

To conclude, we would like to note that the lower densities of hadron matter are needed for percolation (and transition to the deconfined state) in the presence of reflective scattering. It might be useful to note that the rescattering processes are also affected by the reflective scattering. Reflective scattering would lead to noticeable effects at the LHC energies and beyond and could help in searches of the deconfined state and studies of properties of transition mechanism to this state of matter which might proceed by means of percolation. Thus, it will affect description of initial state dynamics in nuclear interactions at the LHC energies by introducing notion of limiting density of strongly interacting matter at respective energies. The appearance of limiting density dependent on energy takes place only at very high energies and has a dynamical origin related to unitarity saturation.

\section{Acknowledgements}

We are grateful to V.A. Petrov for the interesting discussions of reflective scattering mode.

\section{References}

[1] Quark Gluon Plasma. New Discoveries at RHIC: A Case of Strongly Interacting Quark Gluon Plasma. Proceedings, RBRC Workshop, Brookhaven, Upton, USA, May 14-15, 2004: D. Rischke, G. Levin, eds; 2005, 169pp; J. Adams et al. (STAR Collaboration) Nucl. Phys. A 757 (2005)102; K. Adcox et al (PHENIX Collaboration), Nucl. Phys. A 757 (2005) 184.

[2] P. Castorina, K. Redlich, H. Satz. Eur. Phys. J. C 59 (2009) 67, arXiv:0807.4469].

[3] H. Satz, Lecture given at the QGP Winter School, Jaipur, India, Feb. 1, 2008, arXiv:0803.1611.

[4] S.M. Troshin, N.E. Tyurin, Int. J. Mod. Phys. A 22 (2007) 4437.

[5] J.D. Bjorken, Nucl. Phys. Proc. Supl. 25B (1992) 253.

[6] D. Diakonov, [arXiv: hep-ph/0406043], JLAB-THY-04-12, Eur. Phys. J. A 24 (2005) 3; D. Diakonov, V. Petrov, Phys. Lett. B 147 (1984) 351.

[7] T. Goldman, R.W. Haymaker, Phys. Rev. D24 (1981) 724.

[8] S.M. Troshin, N.E. Tyurin, Int. J. Mod. Phys. E 17 (2008) 1619.

[9] S.M. Troshin, N.E. Tyurin, Mod. Phys. Lett. A 23 (2008) 3141. 
[10] J. Cleymans, K. Redlich, H. Satz, E. Suhonen, Z. Phys. C 33 (1986) 151.

[11] P.M. Nadolsky, S.M. Trosnin, N.E. Tyurin, Z. Phys. C 69 (1995) 131. 